\title{
Xkid-ding around
}

During cell division, essential chromosome and microtubule interactions occur that segregate the chromosomes into two sets. This is a tightly controlled process that functions to prevent errors, which may eventually lead to cancer development. One of the main puzzles in cell division has been the identification of the processes that directly control chromosome separation. During metaphase, the sister chromatids line up along the metaphase plate, before separating at the onset of anaphase and moving towards the poles of the spindle. Three microtubule-dependent forces are important during this process of separation, as are microtubule motors such as CENP-E and the kinesin-like motor. Once cells trigger anaphase, certain proteins that maintain sister-chromatid adhesion, such as securin, are targeted for destruction, often through the anaphase-promoting complex (APC). However, there must also be other proteins that have a direct function in the metaphase-anaphase transition, and these have remained unknown.

Recently, Funabiki \& Murray (Cell 102, 411-424; 2000) and Antonio et al. (Cell 102, 425-435; 2000) have identified a new player in anaphase chromosome movement, termed Xkid. Xkid is the Xenopus homologue of human Kid (kinesin-like DNA-binding protein), which is known to be degraded by ubiquitin-mediated proteolysis. Funabiki \& Murray developed a new method to purify metaphase/anaphase chromosomes from Xenopus extracts, and went on to identify proteins that leave these chromsomes at the beginning of the metaphase-anaphase transition. The first protein identified in this manner was Xkid. Antonio et al. identified Xkid in a differential Xenopus messenger-RNA screen.

Xkid localizes along chromosome arms in wild-type extracts. After immunodepletion in these extracts, chromsomes were misaligned along the metaphase plate. The initial stages of spindle assembly are the same as in wild-type extracts but when the chromsomes begin to move towards the poles the chromsomes are abnormally positioned and stretch towards both poles (see picture). Both studies clearly showed that Xkid is usually degraded during anaphase, and Funabiki \& Murray showed that this occurs in a ubiquitin-dependent manner. Overexpression of wild-type or non-degradable Xkid (in an immunodepleted Xkid background) causes the sister chromatids to separate but they can no longer move correctly to the opposite

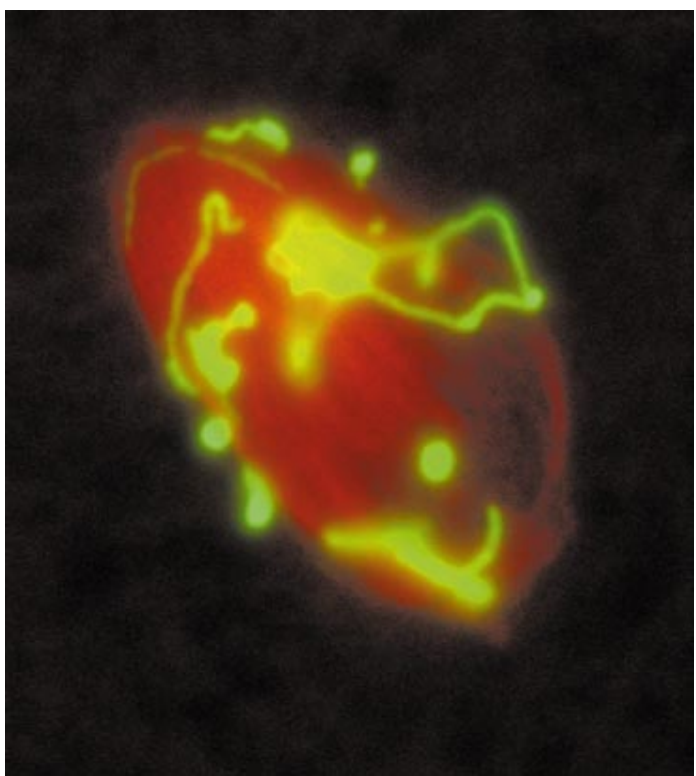

poles of the spindle, hence the conclusion that Xkid is required for chromosome segregation during anaphase.

On the basis of these results, it seems that Xkid has a vital function as a metaphase force that acts to push chromosomes arms apart and towards the poles. The degradation of this protein is essential to allow chromosome movement during anaphase.

This new method of purifying chromosomes from Xenopus egg extracts will allow the identification of further chromsomal proteins, such as those involved in checkpoints. The ability to then deplete these proteins and add wild-type or mutant versions back to the extracts should lead to the widespread use of this system, and to a dramatic increase in our understanding of the metaphase-anaphase transition.

SARAH GREAVES

$\beta$-catenin, p120 associates with the juxtamembrane region of cadherin receptors. Although binding of $\beta$-catenin is essential for the adhesive activity of cadherins, there is some debate over the role of p120 (ref. 11).

Reynolds et al. ${ }^{12}$ previously reported that expression of p120 in fibroblasts induces a 'branching' phenotype. Now members of the same group have shown that these changes in cell shape are abolished by co-expression of activated $\mathrm{Rho}^{2}$. Together with evidence from three different approaches, these results strongly argue that expression of p120 leads to inhibition of Rho.

The study by Noren and colleagues, as well as unpublished work by Bershadsky and colleagues, report the presence of dendrites or enhanced protusion/filopodia after expression of p120 in different cell types. In these cell lines, the phenotype is dependent on activation of Rac and Cdc42 (ref. 3 and A. Bershadsky, personal communication). Inhibition of Rho is also detected biochemically or by a reduced contractility (ref. 3 and A. Bershadsky, personal communication). Thus, it is possible that, after expression of p120, the relative balance between the activities of Rho, Rac and Cdc42 may determine the morphologies that are observed (branches, dendrites or protusions/filopodia). In this setting, however, it is not clear whether Rho inhibition is a consequence of Rac activation ${ }^{13,14}$, or if the two processes occur independently, as proposed by Anastasiadis and colleagues.

Another functional consequence of overexpression of p120 is an increased motility in fibroblasts (A. Bershadsky, personal communication). This enhanced migration is consistent with a requirement for activation of Rac and Cdc42 and inhibition of Rho ${ }^{15}$.
Only pools of p120 present in the cytoplasm can modulate the activity of the small GTPases (refs 2, 3 and A. Bershadsky, personal communication; Fig. 2). In fact, the presence of cadherin receptors can inhibit the morphological changes observed after expression of p120. These results indicate that cadherins can titrate out p120 molecules, reducing their ability to affect RHO signalling. Interestingly, this model is supported by the finding that cadherins containing only the juxtamembrane region in their cytoplasmic tails (the p120binding site) can suppress cell motility ${ }^{16}$.

Although a clear functional interaction between RHO proteins and p120 was shown, no direct binding between these molecules could be detected (refs 2, 3 and A. Bershadsky, personal communication). It is conceivable that the interaction of $\mathrm{p} 120$ with the small GTPases is transient or has a 